\title{
Role of Lipids in the Etiopathogenesis of Periodontal Diseases
}

\section{Balmasova IP ${ }^{1,2}$, Arutyunov SD ${ }^{1}$, Tsarev $\mathrm{VN}^{1}$ and Unanyan $\mathrm{KG}^{3}$ \\ ${ }^{1}$ A.I. Evdokimov Moscow University of Medicine \& Dentistry, Russian Federation \\ ${ }^{2}$ Russian University of Peoples' Friendship, Russian Federation \\ ${ }^{3}$ Dinsk Central Regional Hospital, Krasnodar, Russian Federation}

*Corresponding author: Irina P Balmasova, Head of Laboratory, A.I. Evdokimov Moscow University of Medicine \& Dentistry, Moscow, Russian Federation, Tel: +7 (926) 205-6418;

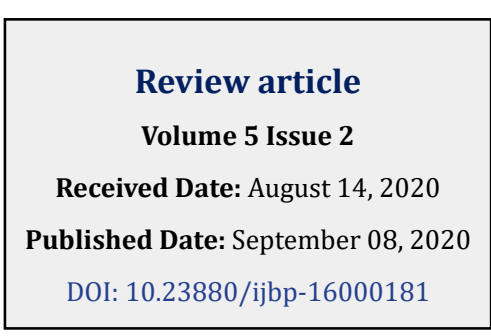

Email: iri.balm@mail.ru

\section{Abstract}

This review article summarizes current knowledge on the association between lipid metabolism and periodontal diseases as well as the role of lipids in the realization of the etiologic and metabolic role of periodontogenic bacteria in this process. Extremely high prevalence of infectious inflammatory periodontal diseases and their currently established impact on the development of systemic conditions is a prerequisite for this paper. The role of lipids as an important factor of the vital activity of micro- and macroorganisms is much addressed in published sources. However, a systemic approach (in particular, in clinical stomatology) is yet to be fully established. Meanwhile, the advances in molecular biology allow for better understanding of the mechanisms of lipid involvement in the development of the virulent properties of periodontogenic bacteria, the modulating the process of bioadgesis of these bacteria to the solid tissues of the oral cavity, their influence on the composition and ultrastructure of the original biofilm of the oral cavity, the specificity of the interaction between periodontitis causative agents and host cells, the regulation of inflammatory and immune processes, pathogenically relevant bone tissue resorption, and the development of systemic effects. As a result, this knowledge may be potentially applied for designing the innovative approaches to the evaluation of the diagnostic markers of pathological periodontal tissues, the treatment and prevention of periodontal diseases.

Keywords: Lipid metabolism; Periodontal disease; Periodontogenic bacteria; Systemic effects

Abbreviations: OMVs: Outer Membrane Vesicles; LPSs: Lipopolysaccharides; MHC: Major Histocompatibility Complex.

\section{Introduction}

Periodontal diseases are characterized by a complex etiopathogenesis and result from a number of factors which lead to periodontal tissue destruction, irreversible bone resorption, and tooth loss [1]. Periodontal diseases are common, i.e., severe periodontitis ranks $6^{\text {th }}$ globally [2]. They have a significant impact on public health due to a high prevalence but are also related to many systemic disorders including diabetes, cardiovascular disorders (i.e., atherosclerosis) etc [3,4]. Given a global burden of periodontitis, the identification of novel therapeutic targets for the treatment and prevention of periodontal diseases is an important issue [5].

Polymicrobial nature of periodontitis is currently recognized. Human inflammatory response has a crucial role in the development and progression of periodontitis thus forcing the researchers to focus on identifying the determinants of the local response to etiologically important bacteria and bacterial products [6]. Recent progress in microbial culture techniques allowed for the identification of about 250 bacterial and fungal species within the dental plaqueswhich can more or less affect the development of periodontitis. The emergence of highly effective methods of molecular biology expanded this list to 750 species [7]. Despite such species diversity, most researchers agree that gram-negative anaerobic bacteria (e.g., Porphyromonas 


\section{International Journal of Biochemistry \& Physiology}

gingivalis, Aggregatibacter actinomycetemcomitans, Tanerella forsythia, Treponema denticola, Fusobacterium nucleatum etc.) are the leading etiology of periodontal tissue damage $[8,9]$. Either of these bacteria have an unique set of virulence factors whose interactions provide the synergism of the periodontogenic effect of emerging microbial associations [10].

Meanwhile, the involvement of general tissue metabolic changes and associated elevation of the levels of proinflammatory mediators (as factors determining the local response to pathogens) are clear. Considering this, systemic conditions related to the development and progression of periodontal diseases are of great interest $[11,12]$.

Among systemic conditions associated with periodontal diseases, lipid metabolism disorders are particularly important $[13,14]$ while hyperlipidemia as a risk factor for the inflammatory destruction of periodontal tissues is inferior only to smoking [15]. Meta-analysis of clinical trials has demonstrated that the development of periodontal diseases is largely associated with low blood levels of high-density lipoproteins and high levels of low-density lipoproteins and cholesterol. Hence, the status of periodontal tissues directly correlates with systemic lipid metabolism [16].

Since the mechanisms of the relationship between lipid metabolism and periodontal diseases as well as the etiologic and metabolic role of periodontogenic bacteria in this process are extensively studied in recent years, this paper aims to analyze the latest available data on this issue.

\section{Lipids and Oral Biofilm Formation}

Biofilm formation on the teeth and gingival mucosa is fundamental to periodontal diseases [17]. The first step is the formation of a pellicle which is largely composed of adsorbed proteins and other macromolecules of the oral cavity (saliva and gingival crevicular fluid) and is different in composition from the oral biofilm/dental plaque $[18,19]$. Pellicle is an acellular layer deposited on solid surfaces which are exposed to oral fluid. It is composed of proteins, glycoproteins, and lipids [20]. Lipids amount one quarter of the dry weight of pellicle and are likely to be of crucial importance for implementing its protective properties though very limited data are now available on the nature of pellicle lipids [17]. Thus, it is known that lipophilic components modulate bioadhesion to the solid tissues of the oral cavity and also the composition and ultrastructure of the initial biofilm of the oral cavity or pellicle, respectively. Lipids on tooth surface can provide pellicle with hydrophobic properties thus preventing bacterial colonization and ultimately reducing the susceptibility to caries. Lipid-enriched pellicle is more resistant to acids that can reduce mineral loss by erosion.
Lipids as fatty acids have anti-inflammatory effects on the soft tissues of the oral cavity [21]. Fatty acid profiles of pellicle samples obtained from various individuals are rather similar [20]. Pellicle contains eleven fatty acids, of them, palmitic $(32 \%)$, stearic $(21 \%)$, oleic $(14 \%)$, erucic $(10 \%)$, and linoleic (5\%) acids are the most common ones [17]. Therefore, lipids are exactly the components which are responsible for the early steps of biofilm formation, i.e., its attachment to tooth surface or oral mucosa.

Classic biofilm life cycle involves several stages, i.e., bacterial attachment and microcolony formation, biofilm growth/maturation, and dispersion. In addition to microorganisms, biofilm contains multiple assemblies of extracellular polysaccharides which enmesh microorganisms to produce diffuse-modifying matrix. Biofilm matrix being a non-cleaved structure greatly affects the chemical and physical microenvironment and species composition of biofilm microbes [22]. Matrix formation is regulated by signaling molecules produced by biofilm bacteria [23]. Over the last decade, it was demonstrated that both structural and protective properties of biofilm matrix are accounted for by exopolysaccharides as well as glycolipids [24].

The role of lipid-containing structures when periodontogenic bacteria (primarily $P$. gingivalis) are integrated into the biofilm should be addressed. This phenomenon was described by Gui MG, et al. [25]. Many gram-negative bacteria are able to generate so-called "outer membrane vesicles" (OMVs), asymmetric singlelayer membrane nanostructures containing virulence factors of these microbes (i.e., toxins or aggression enzymes). A pathogen distantly damage the surrounding bio-environment by releasing OMVs. The initial step of the integration of $P$. gingivalis into the biofilm is the production of OMVs containing gingipains (aggressive proteases) of these bacteria that easily surmount the biofilm due to their small sizes and contribute to the damage of periodontal tissues, the suppression of immune mechanisms, and the invasion of less virulent biofilm constituents before periodontogenic microbes reach epithelial barrier. The formation and separation of OMVs from a bacterial cell require special conditions since vesicle membrane curvature is 14-times greater than external bacterial membrane curvature. As a result, vesicle membrane synthesis requires much greater energy. $P$. gingivalis lipopolysaccharide deacetylation and its anionic fraction release account for greater curvature. The results are the generation of OMVs and the modulation of periodontogenic microbe properties (see below).

\section{Lipids as Virulence Factors of Periodontogenic Bacteria}

Porphyromonas gingivalis is the key periodontal 


\section{International Journal of Biochemistry \& Physiology}

pathogen identified in the biofilm of the gingival sulcus. It is widely recognized that $P$. gingivalis can greatly affect the properties of the whole microbiome of this epitope and ultimately initiate the development of periodontal diseases [26]. These bacteria are among the pathogenic microbes which particularly affect the microecology of periodontal tissues and are a model for studying the etiopathogenesis of periodontal infectious inflammatory conditions [27]. One of the major virulence factors of $P$. gingivalis determining its involvement in periodontal disease pathogenesis and closely related to antibiotic resistance mechanisms are bacterial cell wall lipopolysaccharides (LPSs) [28,29].

LPS is a fundamental structural element of cell membrane of gram-negative bacteria which can induce host innate immune reactions. LPS is composed of three elements, i.e., 0 -antigen, core, and lipid A. O-antigen has a strain-, group-, type-, and variant-specific structure and consists of monosaccharide repeating units. Core includes keto-deoxy-octanoic acid, heptoses, and neutral saccharides (e.g., galactose). Lipid A is an endotoxin which contains two acylated residues of glucose- $\mathrm{N}$-acetyl phosphate $[30,31]$.

Lipid A is the most active biological component that provide LPS with the properties of endotoxin. Lipid A is composed of di-glucosamine with $1^{\prime}$ - and 4'-phosphate residues and attached acyl chains [32,33]. Lipid A structure varies greatly among the species of gram-negative bacteria depending on the differences in the composition of attached fatty acids, the number of phosphorylation sites and replaced groups attached to phosphate residues [34]. However, $P$. gingivalis can deceptively change its lipid structure as a result of dephosphorylation or deacetylation to orchestrate host immune response and to promote chronic inflammation [34-36]. Among heterogeneous patterns of the acetylation of P. gingivalis lipid A, two variants are predominant, i.e., tetraacylated and penta-acylated ones.

Hence, $P$. gingivalis can express two LPS isoforms, i.e., penta-acylated LPS1690 and tetra-acylated LPS1435/1449 which are generated via the changes in lipid A structure in different microecological conditions such as hemin level or culture temperature [35,37]. In fact, Porphyromonas gingivalis cannot produce hemin (which is an important factor of the increased virulence of these bacteria) that, therefore, should be received from the host. Meanwhile, $P$. gingivalis expresses several protein hemin-binding sites which are essential for hemin binding and transport from the host and also have an impact on the formation of lipid A structure [38].

It was demonstrated that LPS1690 and LPS1435/1449 of $P$. gingivalis differentially modulate host immune response, e.g., the expression of human $\beta$-defensin- 2 with its antimicrobial properties, proinflammatory cytokines, and
E-selectin which determines leukocyte adhesion to vascular endothelium [36,38,39]. Thus, it was shown that LPS1690 of $P$. gingivalis can stimulate the expression of specific LPSbinding protein (LBP) by gingival epithelial cells while LPS1435/1449 of $P$. gingivalis lacks these properties $[40,41]$.

LPS/LBP complex interacts with CD14 on monocytes via CD14-binding site. This results in the activation of Tolllike receptor (TLR) 4 [42]. Penta-acylated lipid structures (LPS1690) act as TLR4 agonists while tetra-acylated lipid structures (LPS1435/1449) act as TLR4 antagonists [36,43]. LPS/LBP complex can thereby modulate the expression of proinflammatory cytokines (IL-1, IL-6, and IL-8) by periodontal monocytes induced by various isoforms of $P$. gingivalis LPS [44]. Similar process occurs in gingival fibroblasts [38].

Thismechanismistypical of the LPS of mostgram-negative bacteria although various species of p-Periodontogenic bacteria are characterized by a different set of fatty acids within the lipid A. Thus, $A$. actinomycetemcomitans LPS contains predominantly myristic acid [45] while $F$. nucleatum lipid A contains hexa-acylated lipid A similar to that of Escherichia coli [46]. Structural differences in the LPS of riodontogenic bacteria account for a more potent stimulation of IL-1 $\beta$ secretion under the effect of $F$ nucleatum LPS as compared with P. gingivalis LPS [47].

Meanwhile, unlike other periodontogenic bacteria, $P$. gingivalis LPS is also a potent activator of TLR2 [43]. The nature of this phenomenon was discovered recently. A unique fatty acidic component of $P$. gingivalis lipid A, phosphorylated dihydroceramides (sphingolipid derivatives), is characterized by this activity $[48,49]$.

P. gingivalis synthesizes at least four major ceramides and two of them are selectively adsorbed on damaged tooth surfaces and can penetrate damaged gingival tissues [50]. In addition, these bacteria produce two serine lipids (lipid 654 and lipid 430) which play an important role as inflammatory reaction mediators both in periodontal tissues and other tissues accumulating these lipids [51].

$P$. gingivalis phosphorylated dihydroceramides are currently recognized to facilitate inflammatory reactions and fibroblast morphological changes [52]. These constituents are isolated from the gingival samples of the patients with manifest periodontitis [53]. Phosphorylated dihydroceramides as well as serine dipeptides of $P$. gingivalis interact with TLR2 and stimulate IL-6 production by dendritic cells [54], inhibit osteoblast functions and mineral deposition in bone tissue both in vivo and in vitro [55,56]. As a result, $P$. gingivalis contributes to bone tissue loss in experimental animals and modulates osteoclastogenesis 


\section{International Journal of Biochemistry \& Physiology}

[57].

It was also demonstrated that the lack of sphingolipid synthesis in P. gingivalis leads to the reduced expression of cell-associated arginine- and lysine-gingipains and trypsinlike proteases as well capsule formation by this microbe $[58,59]$.

Therefore, the lipids of periodontogenic bacteria (as well as other prokaryotes) are among their virulence factors. The lipids of most periodontogenic bacteria are endotoxins (lipid A) that can affect host immune system. Their toxic effects and the manner of their interaction with host immune system depend on microecological conditions. The structure of the lipid A of major periodontogenic bacteria can be changed depending on the presence of hemin and temperature. In these conditions, major periodontogenic bacteria demonstrate mainly proinflammatory properties and the ability to induce the resorption of the alveolar ridges of the jaws that results in tooth loss. Moreover, the variability of lipid A structure contributes to the chronicity of periodontal disease course. The production of the structurally different molecules of sphingolipids by major periodontogenic bacteria is less studied. Sphingolipids as virulence factors alter the course of inflammatory reactions, the functions of fibroblasts, osteoblasts, and immune cells, and the production of other virulence factors.

\section{Lipids and the Mechanisms of Periodontogenic Bacteria to Invade Cells}

When discussing the role of lipids in the pathogenesis of periodontal diseases, the mechanism of the interaction between periodontogenic bacteria and microorganism cells should be addressed. Thus, it is known that P. gingivalis being a key periodontogenic pathogen closely contacts and adheres to the epithelial cells of the periodontal pocket while P. gingivalis co-culturing with epithelial cells results in the ultrastructural thickening of fused membranes as P. gingivalis internalizes [60]. This contact allows for delivering bacterial lipids directly to the cellular membranes of host epithelial cells. Another mechanism of the invasion of bacterial lipids into cells can be seen under P. gingivalis interaction with fibroblasts: co-cultured bacterial lipids form lipid films which are ingested by human gingival fibroblasts [61]. P. gingivalis lipids are likely transported in gingival tissue cells either after a close contact with these bacteria or by chemical diffusion of contaminated lipids across the affected tooth surface. Any of these processes can result in the deposition of bacterial lipids in eukaryotic cell membranes thereby exposing cells (including their lipid rafts) to bacterial sphingolipids and serine dipeptide lipids [62].

Cross-contact between host cells and pathogens initiates microbial interactions with signal transduction machinery of infected cells. The major interface of this machinery is lipid rafts [63] and their associated receptors. Lipid rafts are membrane microdomains enriched with cholesterol, sphingolipids, and glycosyl-phosphatidylinositol-anchoring proteins which separate the receptors for different intracellular signaling and transporting processes [64]. Lipid raft formation is related to the ability of sphingolipids and cholesterol to interact mainly which each other that results in their spontaneous separation from other phospholipids of cell membranes. In addition, cholesterol is believed to stabilize lipid rafts by filling the gaps between rather large glycosphingolipids [65].

Cholesterol-enriched membrane microdomains are involved in the induction of both innate and adaptive immunity [66,67]. Lipid rafts are the add-ons to hostpathogen interactions by functioning as the sites for the action of some periodontogenic bacterial toxins $[68,69]$ and the entry of some intracellular pathogens [70]. Lipid rafts act as platforms for protein sorting and signal transduction [64].

P. gingivalis [63,70] and A. actinomycetemcomitans [71] are now recognized to penetrate the lipid rafts of the epithelial cells of periodontal tissues. Bacterial penetration through the lipid rafts has at least two advantages, i.e., the avoidance of intracellular degradation pathway (which results in bacterial degradation) and the induction of intracellular signaling (which results in reduced membrane density and cytoskeletal rearrangement required for bacterial penetration) [70]. Some authors report that internalized lipid rafts cannot easily fuse with lysosomes [72] while cholesterol exhaustion leads to increased $P$. gingivalis localization in association with lysosomes and further pathogen degradation [73].

The penetration of periodontogenic bacteria into epithelial cells through the lipid rafts not only affects the survival of these pathogens but also modulates the functions of epithelial cells. Lipid rafts orchestrate a number of epithelial cell functions, e.g., epithelial barrier function and fighting against bacterial invasion [72]. As demonstrated by the example of $\mathrm{A}$. actinomycetemcomitans, human gingival epithelial cells exposed to the infection with this pathogen contribute to the increase in the levels of proinflammatory cytokines (IL-6 and IL-8) in vitro while TLR4 integration into lipid rafts is a trigger for this process [73].

The results of functional and visualized studies highlight the importance of macrophagic lipid rafts as engulfing and signaling platforms for $P$. gingivalis to facilitate its survival [74]. Recent data demonstrate that pathogenic microorganisms entering the macrophages through the lipid rafts are generally localized in autophagosomes [75]. However, these autophagosomes do not acquire cathepsins 


\section{International Journal of Biochemistry \& Physiology}

required for their generation. As a result, P. gingivalis is able to reproduce in autophagosomes containing non-degraded material [76].

The ability to survive within the macrophages mainly accounts for the systemic effects of periodontogenic bacteria. The presence of alive $P$. gingivalis within the macrophages may be sufficient to allow this microbe for using the migratory potential of macrophages by providing their transfer to other tissues and the infection of other cells that are less resistant to invasion (e.g., endothelial cells). The hypothesis on macrophages as Trojan Horses for the systemic dissemination of $P$. gingivalis is important and should be further developed. Moreover, it was demonstrated that $P$. gingivalis can leave primarily infected host cells and enter new epithelial and endothelial cells to reproduce in them [77].

Therefore, a number of periodontogenic bacteria are intracellular parasites. This property helps penetrate epithelial barrier and migrate to distant organs and tissues by entering macrophages thus providing systemic effects. Periodontogenic bacteria penetrate into host cells through the lipid rafts. This process is largely mediated by sphingolipids produced by these bacteria. This lipid-dependent pathway of intracellular invasion largely promotes the survival of periodontogenic bacteria within the cells.

\section{Lipid Components of Human Biological Media in Infectious Inflammatory Periodontal Diseases}

Lipids are important molecules of the innate immunity in barrier tissues [78]. Thus, saliva contains many lipids including cholesterol, fatty acids, triglycerides, wax esters, cholesterol esters, and squalene. These lipids contribute to various cellular and immune processes including the transport of fat-soluble antioxidants to the mucosal surface and back, mucosal anti-inflammatory and antimicrobial properties $[79,80]$.

Epithelial sphingolipids and short-chain fatty acids are contained in the saliva, corneum stratum of gingival and hard palate epithelium, and mucosal epithelium. These substances demonstrate antimicrobial activity against gram-positive and gram-negative bacteria. These lipids are thought to be the obligatory factors of the innate immune defense against the bacterial infections of barrier tissues [81].

Four potential mechanisms of the realization of the antimicrobial activity of fatty lipids and sphingolipids against bacteria are recognized: (1) membrane destruction mediated by detergent activity; (2) the inclusion of lipids in a bacterial cytoplasmic membrane; (3) the transport of lipids across the bacterial membrane into cytosol; and (4) specific interactions between the lipids and proteins of the bacterial membrane. Fatty acids ultimately result in pore formation in bacterial cytoplasmic membranes, the changes in the structure and functions of cell membrane, cell lysis, and the alterations of various cellular processes either by the interference of the spatial arrangement of molecules or by direct binding with proteins [82]. Thus, bacterial cytoplasmic membranes are the key sites of the realization of lipid activity against $P$. gingivalis that is provided by the incorporation of antimicrobial lipids into these membranes [83].

It was demonstrated that lipid profile of periodontal tissues (as a fatty acid level) determines local inflammatory reactions in infectious inflammatory periodontal diseases [84].It was shown experimentally that saturated fatty acids (e.g., palmitic acid), in contrast to unsaturated fatty acids, induce inflammatory reactions by stimulating the secretion of proinflammatory cytokines (IL- 6 and IL-8) by gingival fibroblasts through the upregulation of surface CD36 as well as the resorption of the alveolar bone in obese mice infected with $P$. gingivalis or exposed to the LPS of A. actinomycetemcomitans [84-86].

Polyunsaturated (omega- 3 and omega-6) fatty acids are the basis for the generation of one of the important classes of molecules regulating inflammation (lipid mediators, or eicosanoids) [87]. Arachidonic acid, eicosapentaenoic acid, and docosahexaenoic acid are the precursors for the biosynthesis of various lipid mediators [88]. These acids are either taken with meals [89] or generated from the phospholipids of cell membranes [90]. There are two pathways of lipid mediator synthesis, i.e., cyclooxygenasemediated and lipoxygenase-mediated ones. These pathways were described 40 years ago and even then were studied with regard to oral disorders [91,92]. More recently, it was hypothesized that some lipid mediators related to oral disorders are the perspective markers of the risk factors of other systemic conditions [93].

The most clinically significant lipid mediators are leukotrienes, prostaglandins, lipoxins, and resolvins [94]. The elevated level of leukotriene B4 in the gingival crevicular fluid clearly correlates with gingival inflammation, the indices of periodontal diseases, and the clinical signs of the loss of tooth attachment to the alveolar bone [95] as demonstrated by experimental studies [96].

Among prostaglandins, the relation with periodontal diseases was demonstrated primarily for prostaglandin E2. Thus, prostaglandin E2 produced by gingival fibroblasts [97] is associated with periodontal diseases and bone tissue loss. As a result, its inhibitors were recommended for 


\section{International Journal of Biochemistry \& Physiology}

periodontitis treatment $[98,99]$.

Lipoxin A4 was recognized as an immunomodulatory agent in periodontal diseases due to its ability to suppress leukocyte involvement in the inflammatory process caused by Porphyromonas gingivalis [100]. Recently, it was demonstrated that lipoxin A4 activates bone tissue regeneration in a pig model of periodontitis [101] and induces the proliferation and migration of human periodontal stem cells [102].

Resolvins are of particular interest. Resolvins are the metabolites of omega-3 polyunsaturated fatty acids which are produced in the course of the inflammatory response being predominant lipid mediators when inflammation resolves $[103,104]$ by limiting the infiltration with leukocytes and the involvement of monocytes in inflammation $[105,106]$. These natural mediators of the resolution of inflammation actively contribute to tissue regeneration and bacterial clearance and enhance (but not inhibit) host defense mechanisms [107].

The analysis of phagocytic cells after resolvin D2 administration demonstrated the increase in circulating neutrophil number, however, neither migration nor accumulation in gingival tissues were observed [108]. This is in line with the observations implying that resolvins prevent the transmigration of these cells along the epithelium $[109,110]$. In contrast, monocyte count reduces while the levels in periodontal tissues increase. However, the phenotype of these macrophages was M2 (resident macrophages) that contributed to the resolution of inflammation [111]. Rapid termination of the local innate immune response and the ability of resolvin D2 to alter dendritic cell maturation via the downregulation of the expression of major histocompatibility complex (MHC) class II molecules account for the reduced efficacy of antigen presentation to $\mathrm{CD}^{+} \mathrm{T}$ cells under the effect of these lipid mediators [112]. This resolvin decreases the production of TNF- $\alpha$ and IFN- $\gamma$ by stimulated human helper $\mathrm{T}$ cells $\left(\mathrm{CD}^{+}\right)$and cytotoxic $\mathrm{T}$ cells $\left(\mathrm{CD}^{+}\right)$. It was also demonstrated that $\mathrm{D}$-series resolvins play a crucial role in $\mathrm{T}$ cell differentiation by preventing the generation of activated Th1 and Th17 which induce inflammation and by boosting the differentiation of regulatory $\mathrm{T}$ cells which exhibit suppressor activity [113].

Experiments with small animals have demonstrated that the control of inflammation and adaptive immune response using resolvins contribute to the prevention and treatment of experimental periodontitis [114-116].

Therefore, periodontal lipids exhibit antimicrobial properties, prevent inflammation and bone resorption, and affect the immune status. Moreover, protective properties of some lipid mediators account for the strong efforts of their potential use in the prevention and treatment for periodontal diseases.

\section{Conclusion}

The role oflipids as components of major periodontogenic bacteria, in the course of the interaction of periodontogenic bacteria with periodontal tissue cells, and as components of human biological media in infectious inflammatory periodontal diseases is clear being highly relevant in terms of pathogenicity. The structure of lipid A (endotoxin) and the ability of periodontogenic bacteria to synthesize sphingolipids with various geometry largely account for the severity of the proinflammatory action of these bacteria, their effects on bone resorption, the ability to produce other virulence factors and to determine infection chronicity.

Sphingolipids of periodontogenic bacteria largely determine how these microbes enter host cells (i.e., epithelial cells, fibroblasts, macrophages etc.) and the ability to migrate to distant organs and tissues. Intracellular invasion is mediated by sphingolipid integration into cell lipid rafts followed by pathogen survival within the endosomes that do not destroy bacteria. Host lipid metabolism largely determines the resistance to microbial invasion, the severity of inflammation, the type of immune reactions, and the ability of periodontal tissues to counteract their dysfunctions.

In conclusion, lipid metabolism in periodontogenic bacteria and host organism (both local and systemic ones) is important for the development and progression of periodontal diseases. More research in this area holds promise for the advances in clinical periodontology and the design of innovative preventive and therapeutic modalities for these common conditions that have systemic effects.

\section{References}

1. Hajishengallis G, Darveau RP, Curtis MA (2012) The keystone-pathogen hypothesis. Nat Rev Microbiol 10(10): 717-725.

2. Tonetti MS, Jepsen S, Jin L, Otomo-Corgel J (2017) Impact of the global burden of periodontal diseases on health, nutrition and wellbeing of mankind: A call for global action. J Clin Periodontol 44(5): 456-462.

3. Bui FQ, Almeida-da-Silva CLC, Huynh B, Trinh A, Liu J, et al. (2019) Association between periodontal pathogens and systemic disease. J Biomed Sci 42(1): 27-35.

4. Nakao R, Hasegawa H, Dongying B, Ohnishi M, Senpuku $\mathrm{H}$ (2016) Assessment of outer membrane vesicles of periodontopathic bacterium Porphyromonas gingivalis as possible mucosal immunogen. Vaccine 34 (38): 4626- 


\section{International Journal of Biochemistry \& Physiology}

4634.

5. de Andrade KQ, Almeida-da-Silva CLC, Coutinho-Silva $\mathrm{R}$ (2019) Immunological pathways trriggered by Porphyromonas gingivalis and Fusobacterium nucleatum: therapeutic possibilities? Mediators Inflamm 2019: 7241312 .

6. Cekici A, Kantarci A, Hasturk H, Van Dyke TE (2014) Inflammatory and immune pathways in the pathogenesis of periodontal disease. Periodontol 2000 64(1): 57-80.

7. Al-hebshi NN, Shuga-Aldin HM, Al-Sharabi AK, Ghandour I (2014) Subgingival periodontal pathogens associated with chronic periodontitis in Yemenis. BMC Oral Health 14(13).

8. Nikolayeva EN, Tsarev VN, Ippolitov EV (2011) Periodontopathogenicbacterias as indicators of risk of occurrence and development of periodontitis (part 2). Dentistry 4: 4-7

9. Tsarev VN (2013) Microbiology, virology and oral immunology. Geotar-Media, Moscow, Russian Federation, pp: 576.

10. Wolcott R, Costeryon JW, Raoult D, Cutler SJ (2013) The polymicrobial nature of biofilm infection. ClinMicrobiol Infect 19(2): 107-112.

11. Tsepov LM, Nikolaev AI, Tsepov EL, Tsepov AL (2009) Periodontal pathology in systemic diseases. Maestro dentistry 1: 64-67.

12. Hasturk H, Kantarci A, Van Dyke TE (2012) Oral inflammatory diseases and systemic inflammation: role of the macrophage. Front Immunol 3: 118-134.

13. Muluke M, Gold T, Kiefhaber K, Al-Sahli A, Celenti R, et al. (2016) Diet-induced obesity and its differential impact on periodontal bone loss. J Dent Res 95(2): 223-229.

14. Chaffee BW, Weston SJ (2010) Association between chronic periodontal disease and obesity: a systematic review and meta-analysis. J Periodontol 81(12): 17081724.

15. Nishida N, Tanaka M, Hayashi N, Nagata H, Takeshita T, et al. (2005) Determination of smoking and obesity as periodontitis risks using the classification and regression tree method. J Periodontol 76(6): 923-928.

16. Nepomuceno R, Pigossi SC, Finoti LS, Orrico SRP, Cirelli JA, et al. (2017) Serum lipid levels in patients with periodontal disease: A meta-analysis and metaregression. J ClinPeriodontol 44(12): 1192-1207.
17. Reich M, Hanning C, AL-Ahmad A, Bolek R, Kummerer K (2012) A comprehensive method for determination of fatty acids in the initial oral biofilm (pellicle). J Lipid Res 53(10): 2226-2230.

18. Hannig C, Hannig M (2009) The oral cavity-a key system to understand substratum-dependent bioadhesion on solid surfaces in man. Clin Oral Investig 13(2): 123-139.

19. Hannig M, Joiner A (2006) The structure, function and properties of the acquired pellicle. Monogr Oral Sci 19: 29-64.

20. Reich M, Kummerer K, AL-Ahmad A, Hannig C (2013) Fatty acid profile of the initial oral biofilm (pellicle): an in-situ study. Lipids 48(9): 929-937.

21. Kensche A, Reich M, Kummerer K, Hannig M, Hannig C (2013) Lipids in preventivedentistry. Clin Oral Investig 17(3): 669-685.

22. Hentzer M, Teitzel GM, Balzer GJ,Heydorn A, Molin S, et al. (2001) Alginate overproduction affects Pseudomonas aeruginosa biofilm structure and function. J Bacteriol 183(18): 5395-5401.

23. Tolker-Nielsen T (2015) Biofilm development. Microbiol Spectr 3(2): MB-0001-2014.

24. Sallans L, Giner JL, Kiemle DJ, Custer JE, Kaneshiro ES (2013) Structural identities of four glycosylated lipids in the oral bacterium Streptococcus mutans UA159. Biochim Biophys Acta 1831(7): 1239-1249.

25. Gui MJ, Dashper SG, Slakeski N, Chen YY, Reynolds EC (2016) Spheres of influence: Porphyromonasgingivalis outer membrane vesicles. Mol Oral Microbiol 31(5): 365-378.

26. Hajishengallis G, Liang S, Payne MA, Hashim A, Jotwani $\mathrm{R}$, et al. (2011) Low-abundance biofilm species orchestrates inflammatory periodontal disease through the commensal microbiota and complement. Cell Host \& Microbe 10(5): 497-506.

27. Yanushevich 00, Akhmedov GD, Panin AM, Arutyunov SD, Tsarev VN (2019) Oral microecology and infectiousinflammatory complications in surgical dentistry. Practical Medcine, Moscow, Russian Federation, pp: 192.

28. Darveau RP (2009) The oral microbial consortium's interaction with the periodontal innate defense system. DNA Cell Bio 28(8): 389-395.

29. Varbanets LD (2016) Structure, function and biological activity of lipopolysaccharide lipid A. Mikrobiol Z 78(6): 37-49. 


\section{International Journal of Biochemistry \& Physiology}

30. Amor K, Heinrichs DE, Frirdich E, Ziebell K, Johnson RP, et al. (2000) Distribution of core oligosaccharide types in lipopolysaccharides from Escherichia coli. Infect Immun 68(3): 1116-1124.

31. Trent MS (2004) Biosynthesis, transport, and modification of lipid A. Biochem Cell Biol 82(1): 71-86.

32. Raetz CR, Guan Z, Ingram BO, Six DA, Song F, et al. (2009) Discovery of new biosynthetic pathways: the lipid A story. J Lipid Res 50: 103-108.

33. Raetz CR, Reynolds CM, Trent MS, Bishop RE (2007) Lipid A modification systems in gram-negative bacteria. Annu Rev Biochem 76: 295-329.

34. Al-Qutub MN, Braham PH, Karimi-Naser LM, Liu X, Genco CA, et al. (2006) Hemin-dependent modulation of the lipid A structure of Porphyromonas gingivalis lipopolysaccharide. Infect Immun 74(8): 4474-4485.

35. Reife RA, Coats SR, Al-Qutub M, Dixon DM, Braham PA, et al. (2006) Porphyromonas gingivalis lipopolysaccharide lipid A heterogeneity: differential activities of tetraand penta-acylated lipid A structures on E-selectin expression and TLR4 recognition. Cell Microbiol 8(5): 857-868.

36. Curtis MA, Percival RS, Devine D, Darveau RP, Coats SR, et al. (2011) Temperature-dependent modulation of Porphyromonas gingivalis lipid A structure and interaction with the innate host defenses. Infect Immun 79(3): 1187-1193.

37. Rangarajan M, Aduse-Opoku J, Paramonov NA, Hashim A, Curtis MA (2017) Hemin binding by Porphyromonas gingivalis strains is dependent on the presence of A-LPS. Mol Oral Microbiol 32(5): 365-374.

38. Herath TD, Wang Y, Seneviratne CJ, Lu Q, Darveau RP, et al. (2011) Porphyromonas gingivalis lipopolysaccharide lipid A heterogeneity differentially modulates the expression of IL-6 and IL-8 in human gingival fibroblasts. J ClinPeriodontol 38(8): 694-701.

39. Lu Q, Darveau RP, Samaranayake LP, Wang CY, Jin LJ (2009) Differential modulation of human \{beta\}defensins expression in human gingival epithelia by Porphyromonas gingivalis lipopolysaccharide with tetraand penta-acylated lipid A structures. Innate Immun 15(6): 325-335.

40. Ding PH, Wang CY, Darveau RP, Jin LJ (2013) Porphyromonas gingivalis LPS stimulates the expression of LPS-binding protein in human oral keratinocytes in vitro. Innate Immun 19(1): 66-75.
41. Ding PH, Wang CY, Darveau RP, Jin LJ (2013) Nuclear factor- $\mathrm{KB}$ and p38 mitogen-activated protein kinase signaling pathways are critically involved in Porphyromonas gingivalis lipopolysaccharide induction of lipopolysaccharide-binding protein expression in human oral keratinocytes. Mol Oral Microbiol 28(2): 129-141.

42. Freudenberg MA, Tchaptchet $S$, Keck S, Fejer G, Huber M, et al. (2008) Lipopolysaccharide sensing an important factor in the innate immune response to Gram-negative bacterial infections: benefits and hazards of LPS hypersensitivity. Immunobiology 213(3-4): 193-203.

43. Darveau RP, Pham TT, Lemley K, Reife RA, Bainbridge BW, et al. (2004) Porphyromonas gingivalis lipopolysaccharide contains multiple lipid A species that functionally interact with both toll-like receptors 2 and 4. Infect Immun 72(9): 5041-5051.

44. Ding PH, Darveau RP, Wang CY, Jin L (2017) 3LPSbinding protein and its interactions with $P$. gingivalis LPS modulate pro-inflammatory response and Toll-like receptor signaling in human oral keratinocytes. PLoS One 12(4): e0173223.

45. Brondz I, Olsen I (1984) Determination of acids in whole lipopolysaccharide and in free lipid A from Actinobacillus actinomycetemcomitans and Haemophilus aphrophilus. J Chromatogr 308: 19-29.

46. Asai Y, Makimura Y, Kawabata A, Ogawa T (2007) Soluble CD14 discriminates slight structural differences between lipid as that lead to distinct host cell activation. J Immunol 179(11): 7674-7683.

47. Taxman DJ, Swanson KV, Broglie PM, Wen H, Holley Guthrie E, et al. (2012) Porphyromonas gingivalis mediates inflammasome repression in polymicrobial cultures through a novel mechanism involving reduced endocytosis. J BiolChem 287(39): 32791-32799.

48. Nichols FC, Bajrami B, Clark RB, Housley W, Yao X (2012) Free lipid A isolated from Porphyromonas gingivalis lipopolysaccharide is contaminated with phosphorylated dihydroceramide lipids: Recovery in diseased dental samples. Infect Immun 80(2): 860-874.

49. Nichols FC, Yao X, Bajrami B, Downes J, Finegold SM, et al. (2011) Phosphorylated Dihydroceramides from Common Human Bacteria Are Recovered in Human Tissues 6(2): e16771.

50. Nichols FC (1998) Novel ceramides recovered from Porphyromonas gingivalis: Relationship to adult periodontitis. J Lipid Res 39(12): 2360-2372. 


\section{International Journal of Biochemistry \& Physiology}

51. ClarkRB, Cervantes JL, Maciejewski MW, Farrokhi $\mathrm{V}$, Nemati R, et al. (2013) Serine lipids of Porphyromonasgingivalis are human and mouse Toll-like receptor 2 ligands. Infect Immun 81(9): 3479-3489.

52. Nichols FC, Riep B, Mun J, Morton MD, Bojarski MT, et al. (2004) Structures and biological activity of phosphorylated dihydroceramides of Porphyromonas gingivalis. J Lipid Res 45(12): 2317-2330.

53. Nichols FC (1994) Distribution of 3-hydroxy iC17:0 in subgingival plaque and gingival tissue samples: relationship to adult periodontitis. Infect Immun 62(9): 3753-3760.

54. Nichols FC, Housley WJ, O'Conor CA, Manning T, Wu $S$, et al. (2009) Unique lipids from a common human bacterium represent a new class of Toll-like receptor 2 ligands capable of enhancing autoimmunity. Am J Pathol 175(6): 2430-2438.

55. Olsen I, Nichols FC (2018) Are sphingolipids and serine dipeptide lipids underestimated virulence factors of Porphyromonas gingivalis? Infect Immun 86(7): e0003518.

56. Wang YH, Jiang J, Zhu Q, AlAnezi AZ, Clark RB, et al. (2010) Porphyromonas gingivalis lipids inhibit osteoblastic differentiation and function. Infect Immun 78(9): 37263735.

57. Zhang P, Liu J, Xu Q, Harber G, Feng X, et al. (2011) TLR2-dependent modulation of osteoclastogenesis by Porphyromonas gingivalis through differential induction of NFATc1 and NF-\{kappa\}B. J BiolChem 286(27): 2415924169.

58. Bainbridge BW, Hirano T, Grieshaber N, Davey ME (2015) Deletion of a 77-base-pair inverted repeat element alters the synthesis of surface polysaccharides in Porphyromonas gingivalis. J Bacteriol 197(7): 12081220.

59. Moye ZD, Valiuskyte K, Dewhirst FE, Nichols FC, Davey ME (2016) Synthesis of sphingolipids impacts survival of Porphyromonasgingivalis and the presentation of surface polysaccharides. Front Microbiol 7: 1919.

60. Sandros J, Papapanou P, Dahlen G (1993) Porphyromonas gingivalis invades oral epithelial cells in vitro. J Periodontal Res 28(3): 219-226.

61. Nichols FC, Levinbook H, Shnaydman M, Goldschmidt J (2001) Prostaglandin E2 secretion from gingival fibroblasts treated with interleukin-1beta: effects of lipid extracts from Porphyromonasgingivalis or calculus.
J Periodontal Res 36(3): 142-152.

62. Olsen I, Nichols FC (2018) Are sphingolipids and serine dipeptide lipids underestimated virulence factors of Porphyromonasgingivalis? Infect Immun 86(7): e0003518.

63. Simons K, Toomre D (2000) Lipid rafts and signal transduction. Nat Rev Mol Cell Biol 1(1): 31-39.

64. Simons K., Ikonen E (1997) Functional rafts in cell membranes. Nature 387(6633): 569-572.

65. Riethmuller J, Riehle A, Grassme H, Gulbins E (2006) Membrane rafts in host-pathogen interactions. Biochim Biophys Acta 1758(12): 2139-2147.

66. Pizzo P, Viola A (2003) Lymphocyte lipid rafts: structure and function. Curr Opin Immunol 15(3): 255-260.

67. Triantafilou M, Brandenburg K, Gutsmann T, Seydel U, Triantafilou K (2002) Innate recognition of bacteria: engagement of multiple receptors. Crit Rev Immunol 22(4): 251-268.

68. Boesze Battaglia K, Besack D, McKay T, Zekavat A, Otis L, et al. (2006) Cholesterol-rich membrane microdomains mediate cell cycle arrest induced by Actinobacillus actinomycetemcomitans cytolethal-distending toxin. Cell Microbiol 8(5): 823-836.

69. Fong KP, Pacheco CM, Otis LL, Baranwal S, Kieba IR, et al. (2006) Actinobacillus actinomycetemcomitans leukotoxin requires lipid microdomains for target cell cytotoxicity. Cell Microbiol 8(11): 1753-1767.

70. Manes S, del Real G, Martinez AC (2003) Pathogens: raft hijackers. Nat Rev Immunol 3(7): 557-568.

71. Imai H, Fujita T, Kajiya M, Ouhara K, Yoshimoto T, et al. (2016) Mobilization of TLR4 into lipid rafts by Aggregatibacter actinomycetemcomitans in gingival epithelial cells. Cell Physiol Biochem 39(5): 1777-1786.

72. Simons K, Gruenberg J (2000) Jamming the endosomal system: lipid rafts and lysosomal storage diseases. Trends Cell Biol 10(11): 459-462.

73. Wang M, Hajishengallis G (2008) Lipid raft-dependent uptake, signaling, and intracellularfate of Porphyromonas gingivalis in mouse macrophages. Cell Microbiol 10(10): 2029-2042.

74. Saito A, Kokubu E, Inagaki S, Imamura K, Kita D, et al. (2012) Porphyromonas gingivalis entry into gingival epithelial cells modulated by Fusobacterium nucleatumis dependent on lipid rafts. Microb Pathog 53: 234-242. 


\section{International Journal of Biochemistry \& Physiology}

75. Amer AO, Byrne BG, Swanson MS (2005) Macrophages rapidly transfer pathogens from lipid raft vacuoles to autophagosomes. Autophagy 1(1): 53-58.

76. Dorn BR, Dunn WA, Progulske Fox A (2002) Bacterial interactions with the autophagic pathway. Cell Microbiol 4(1): 1-10.

77. Li L, Michel R, Cohen J, Decarlo A, Kozarov E (2008) Intracellular survival and vascular cell-to-cell transmission of Porphyromonas gingivalis. BMC Microbiol 8: 26.

78. 78. Drake DR, Brogden KA, Dawson DV, Wertz PW (2008) Thematic review series: skin lipids. Antimicrobial lipids at the skin surface. J Lipid Res 49(1): 4-11.

79. Brasser A, Barwacz C, Bratt CL, Dawson D (2011) Free sphingosine in human saliva. J Dent Res 90: 3465.

80. Brasser AJ, Barwacz CA, Dawson DV, Brogden KA, Drake DR, et al. (2011) Presence of wax esters and squalene in human saliva. Arch Oral Biol 56(6): 588-591.

81. Gorr SU (2012) Antimicrobial peptides in periodontal innate defense. Front Oral Biol 15: 84-98.

82. Desbois AP, Smith VJ (2010) Antibacterial free fatty acids: activities, mechanisms of action and biotechnological potential. Appl Microbiol Biotechnol 85(6): 1629-1642.

83. Fischer CL, Walters KS, Drake DR, Dawson DV, Blanchette DR, et al. (2013) Oral mucosal lipids are antibacterial against Porphyromonasgingivalis, induce ultrastructural damage, and alter bacterial lipid and protein compositions. Int J Oral Sci 5(3): 130-140.

84. Shikama Y, Kudo Y, Ishimaru N, Funaki M (2017) Potential role of free fatty acids in the pathogenesis of periodontitis and primary Sjögren's syndrome. Int J Mol Sci 18(4): 836-843.

85. Muluke M, Gold T, Kiefhaber K, Al-Sahli A, Celenti R, et al. (2016) Diet-induced obesity and its differential impact on periodontal bone loss. J Dent Res 95(2): 223-229.

86. Lu Z, Li Y, Brinson CW, Kirkwood KL, Lopes-Virella MF, Huang Y (2017) CD36 is upregulated in mice with periodontitis and metabolic syndrome and involved in macrophage gene upregulation by palmitate Oral Dis 23(2): 210-218.

87. Sommakia S, Baker OJ (2017) Regulation of inflammation by lipid mediators in oral diseases. Oral Dis 23(5): 576597.

88. Tessaro FH, Ayala TS, Martins JO (2015) Lipid mediators are critical in resolving inflammation: a review of the emerging roles of eicosanoids in diabetes mellitus. Bio Med Res Int 2015: 568408.

89. Astudillo AM, Balgoma D, Balboa MA, Balsinde J (2012) Dynamics of arachidonic acid mobilization by inflammatory cells. Biochim Biophys Acta 1821(2): 249256.

90. Serini S, Piccioni E, Merendino N, Calviello G (2009) Dietary polyunsaturated fatty acids as inducers of apoptosis: implications for cancer. Apoptosis 14(2): 135152.

91. Pace-Asciak C, Grastrom E (1983) Prostaglandins and related substances. Elsevier Publishing Company, Amsterdam, New York, Oxford, pp: 229.

92. Samuelson B (1986) Leukotrienes and other lipoxygenase products. Prog Lipid Res 25(1-4): 13-18.

93. Offenbacher S, Odle BM, Braswell LD, Johnson HG, Hall CM, et al. (1989) Changes in cyclooxygenase metabolities in experimental periodontitis in Macacamulatta. J Periodont Res 24(1): 63-74.

94. Back M, Airila-Mansson S, Jogestrand T, Soder B, Soder PÖ (2007) Increased leukotriene concentrations in gingival crevicular fluid from subjects with periodontal disease and atherosclerosis. Atherosclerosis 193(2): 389-394.

95. Pradeep A, Manjunath S, Swati PP, Shikha C, Sujatha PB (2007) Gingival crevicular fluid levels of leukotriene B4 in periodontal health and disease. J Periodontol 78(12): 2325-2330.

96. Busch L, Miozza V, Sterin-Borda L, Borda E (2009) Increased leukotriene concentration $\times$ in submandibular glands from rats with experimental periodontitis. Inflam Res 58(7): 423-430.

97. Nichols FC, Levinbook H, Shnaydman M, Goldschmidt J (2001) Prostaglandin E2 secretion from gingival fibroblasts treated with interleukin-1beta: effects of lipid extracts from Porphyromonas gingivalis or calculus. J Periodontal Res 36(3): 142-152.

98. Yamaguchi M, Kasai K (2005) Inflammation in periodontal tissues in response to mechanical forces. Arch Immunol Ther Exp (Warsz) 53(5): 388-398.

99. Noguchi K, Miyauchi M, Oka H, Komaki M, Somerman MJ, et al. (2007) Cyclooxygenase-2-dependent prostaglandin E2 upregulates interleukin (IL)- $1 \alpha$-induced IL-6 generation in mouse cementoblasts. J Periodontol 78(1): 135-140. 


\section{International Journal of Biochemistry \& Physiology}

100. Pouliot M, Clish CB, Petasis NA, Van Dyke TE, Serhan CN (2000) Lipoxin A4 analogues inhibit leukocyte recruitment to Porphyromonas gingivalis: A role for cyclooxygenase-2 and lipoxins in periodontal disease. Biochemistry 39(16): 4761-4768.

101. Van Dyke TE, Hasturk H, Kantarci A, Freire MO, Nguyen D, et al. (2015) Proresolving nanomedicines activate bone regeneration in periodontitis. J Dent Res 94(1): 148-156.

102. Cianci E, Recchiuti A, Trubiani O, Diomede F, Marchisio M, et al. (2016) Human periodontal stem cells release specialized proresolving mediators and carry immunomodulatory and prohealing properties regulated by lipoxins. Stem Cells Transl Med 5(1): 20-32.

103. Buckley CD, Gilroy DW, Serhan CN (2014) Proresolving lipid mediators and mechanisms in the resolution of acute inflammation. Immunity 40(3): 315327.

104. Serhan CN, Hong S, Gronert K, Colgan SP, Devchand PR, et al. (2002) Resolvins: a family of bioactive products of omega-3 fatty acid transformation circuits initiated by aspirin treatment that counter proinflammation signals. J Exp Med 196(8): 1025-1037.

105. Chiurchiu V, Leuti A, Maccarrone M (2018) Bioactive lipids and chronic inflammation: managing the fire within. Front Immunol 9: 38.

106. Serhan CN (2014) Pro-resolving lipid mediators are leads for resolution physiology. Nature 510(7503): 92101.

107. Serhan CN, Chiang N, Van Dyke TE (2008) Resolving inflammation: dual anti-inflammatory and proresolution lipid mediators. Nat Rev Immunol 8(5): 349361.

108. Mizraji G, Heyman O, Van Dyke TE, Wilensky A (2018) Resolvin D2 restrains Th1 immunity and prevents alveolar bone loss in murine periodontitis. Front Immunol 9: 785-794.

109. Chiang N, Dalli J, Colas RA, Serhan CN (2015) Identification of resolvin D2 receptor mediating resolution of infections and organ protection. J Exp Med 212(8): 1203-1217.

110. Serhan CN, Clish CB, Brannon J, Colgan SP, Chiang $\mathrm{N}$, et al. (2000) Novel functional sets of lipid-derived mediators with antiinflammatory actions generated from omega-3 fatty acids via cyclooxygenase 2-nonsteroidal antiinflammatory drugs and transcellular processing. J Exp Med 192(8): 1197-1204.

111. Pope NH, Salmon M, Davis JP, Chatterjee A, Su G, et al. (2016) D-series resolvins inhibit murine abdominal aortic aneurysm formation and increase M2 macrophage polarization. FASEB J 30(12): 4192-4201.

112. Serhan CN, Petasis NA (2011) Resolvins and protectins in inflammation resolution. Chem Rev 111(10): 5922-5943.

113. Chiurchiu V, Leuti A, Dalli J, Jacobsson A, Battistini L, et al. (2016) Proresolving lipid mediators resolvin D1, resolvin D2, and maresin 1 are critical in modulating $\mathrm{T}$ cell responses. SciTransl Med 8(353): 353.

114. Gao L, Faibish D, Fredman G, Herrera BS, Chiang N, et al. (2013) Resolvin E1 and chemokine-like receptor 1 mediate bone preservation. J Immunol 190(2): 689-694.

115. Hasturk H, Kantarci A, Goguet-Surmenian E, Blackwood A, Andry C, et al. (2007) Resolvin E1 regulates inflammation at the cellular and tissue level and restores tissue homeostasis in vivo. J Immunol 179(10): 70217029 .

116. Lee CT, Teles R, Kantarci A, Chen T, McCafferty J, et al. (2016) Resolvin E1 reverses experimental periodontitis and dysbiosis. J Immunol 197(7): 2796-2806. 